\title{
Laparoscopic liver resection with lymph node dissection for gallbladder tumors suspected to be T1b/T2 carcinoma
}

\author{
Masashi Isetani', Zenichi Morise ${ }^{2}$, Akihiko Horiguchi' \\ ${ }^{1}$ Department of Surgery, Fujita Health University Banbuntane Houtokukai Hospital, Nagoya, Aichi 454-8509, Japan. \\ ${ }^{2}$ Department of Surgery, Fujita Health University School of Medicine, Toyoake, Aichi 470-1192, Japan.
}

Correspondence to: Dr. Zenichi Morise, Department of Surgery, Fujita Health University School of Medicine, 1-98 Dengakugakubo Kutsukakecho, Toyoake, Aichi 470-1192, Japan. E-mail: zmorise@fujita-hu.ac.jp

How to cite this article: Isetani M, Morise Z, Horiguchi A. Laparoscopic liver resection with lymph node dissection for gallbladder tumors suspected to be T1b/T2 carcinoma. Hepatoma Res 2017;3:170-7.
Article history:
Received: 02-05-2017
Accepted: 21-07-2017
Published: 09-08-2017

\section{Key words:}
Gallbladder carcinoma,
laparoscopic surgery,
laparoscopic liver resection,
lymph node dissection

\begin{abstract}
Aim: The short-term perioperative results of laparoscopic treatment of gallbladder (GB) carcinoma were evaluated to determine whether this technique can be a feasible treatment option. Methods: Ten patients with fundus/body GB tumors (GBTs) underwent laparoscopic liver resection (LLR) and lymph node dissection. Additionally, 124 patients underwent LLR for liver tumors. These 124 LLRs included 79 partial resections (PRs), 11 left lateral sectionectomies (LLSs), 25 anatomical resections (ARs), and 9 small ARs (SARs). The operation time (OT), intraoperative blood loss (BL), and postoperative length of hospital stay (LOS) were compared between the GBT and various LLR groups. Results: The median (range) OT in the GBT, PR, LLS, AR, and SAR groups was 298 (186-488), 245 (84-700), 328 (150-682), 458 (224-848), and 352 (274-696) min, respectively. The BL was 109 (10-500), 50 (0/uncountable-3,270), $100(10-516), 375$ (25-3,569), and 705 (35-1,920) $\mathrm{mL}$, respectively. The LOS was 16 (8-105), 15 (5-254), 13 (11-52), 22 (8-44), and 15 (8-44) days, respectively. The OT and BL were significantly different between the GBT and AR groups. Conclusion: Laparoscopic surgery could be a good treatment option for GBTs suspected to be T1b/T2 GB carcinoma in the GB body/fundus without cystic duct invasion.
\end{abstract}

\section{INTRODUCTION}

Since the development of laparoscopic liver resection (LLR) in the early 1990s,,$^{[1-3]}$ this technique has rapidly expanded from partial LLR of the easily accessible anterolateral segments [segment 2 (S2), S3, S4b, S5, and S6] to left lateral sectionectomy (LLS), hemihepatectomy, other sectionectomies, segmentectomies and resections of S7, S8, and S1, and more complicated limited or modified anatomical LLRs. ${ }^{[4]}$ LLR has recently become widely accepted as a less invasive treatment for liver tumors with specific advantages such as less intraoperative bleeding and a shorter postoperative length of hospital stay (LOS). ${ }^{[5,6]}$ Partial resection (PR) of the anterolateral segments is currently considered a standard procedure..$^{[5]}$

Few reports have described intended laparoscopic

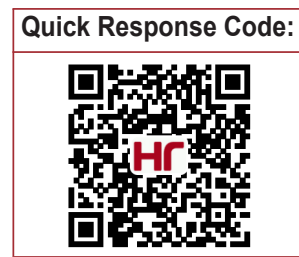


treatments for gallbladder carcinoma (GBC):[7-9] however, many studies of occult GBC revealed high incidences of port site recurrence and peritoneal dissemination after laparoscopic cholecystectomy. ${ }^{[10,11]}$ The treatment of T1b/T2 GBC, ${ }^{[12]}$ which is not in the early stage of intramucosal layer but without serosal invasion, involves a combination of liver resection, lymph node ( $\mathrm{LN})$ dissection, and bile duct resection and reconstruction in cases of invasion. ${ }^{[13,14]}$ Laparoscopic procedures have been less commonly adapted to GBC treatment mainly because of concerns regarding the aggressive features of the malignancy and the technically demanding surgical procedure. ${ }^{[7-9]}$ However, the liver resection technique performed for treatment of $T 1 \mathrm{~b} / T 2 \mathrm{GBC}$ involves resection of either the GB bed or $S 4 b+5+6 a$, both of which require resection of the anterolateral segments. $L N$ dissection has also been applied to other more popular procedures. ${ }^{[15]}$ Because bile duct resection and reconstruction is not necessary during surgical treatment of T1b/T2 GBC of the body/ fundus without cystic duct invasion, the operation is a simple combination of anterolateral LLR and limited $\mathrm{LN}$ dissection. Furthermore, tumor dissemination and port site recurrence are thought to occur mainly due to bile leakage from intraoperative GB perforation. ${ }^{[9,11]}$ Theoretically, combined resection of the GB bed liver could prevent these events. ${ }^{[10,11,16,17]}$ Therefore, we have employed a laparoscopic procedure for treatment of GB tumors (GBTs) suspected to be $T 1 \mathrm{~b} /$ T2 GBC located in the GB body/fundus without cystic duct invasion.

In this study, to determine whether laparoscopic treatment of $T 1 \mathrm{~b} / \mathrm{T} 2 \mathrm{GBC}$ is a feasible treatment option, we compared the short-term results of patients who underwent this procedure and those of patients who underwent various types of LLR.

\section{METHODS}

Among 28 patients who underwent GB resection for suspected GBC from November 2011 to June 2015, 10 patients with GBTs suspected to be T1b/T2 GBC in the GB fundus/body underwent LLR and LN dissection. The other patients underwent laparoscopic full-thickness cholecystectomy for suspected T1a GBC or open surgery for suspected $\geq \mathrm{T} 3 \mathrm{GBC}$ or possible bile duct resection based on preoperative assessment.

Three patients with T2 GBC underwent LLR of $S 4 b+5+6 a$ with regional $L N$ dissection, and the other seven patients underwent LLR of the GB bed liver with peri-cystic duct LN and peri-bile duct $L N$ dissection. The patients' data are shown in Table 1.

In total, 124 patients underwent LLR for liver tumors (80 hepatocellular carcinomas, 35 metastatic tumors, and 9 others). These 124 LLR procedures included 79 PRs, 11 LLSs, 25 anatomical resections (ARs) (resection of one or more segments, excluding LLS), and 9 small ARs (SARs) (resection of less than a full segment and sometimes combined resection of those).

The conversion, morbidity, and mortality rates were compared between the GBT and various LLR groups. The perioperative short-term results [operation time (OT), intraoperative blood loss (BL), and postoperative LOS] of the 10 patients with GBTs were compared with those of the patients who underwent various types of LLR (PR, LLS, AR, and SAR).

Patients were fully involved in the treatment decision-making process. Informed consent was obtained from each patient for both treatment and use of data in the study. The data obtained through the medical record review were managed according to the privacy policy and ethics code of our institute. The surgeries were performed with the permission of our hospital review board.

\section{Statistical analysis}

Results are expressed as median (range) and mean \pm

Table 1: Characteristics of the 10 patients who underwent laparoscopic surgery for suspected T1b/T2 GBC

\begin{tabular}{|c|c|c|c|c|c|c|c|c|c|c|}
\hline Gender & $\begin{array}{c}\text { Age } \\
\text { (years) }\end{array}$ & Child-Pugh & $\begin{array}{l}\text { T-stage } \\
\text { (clinical) }\end{array}$ & $\begin{array}{c}\text { T-stage } \\
\text { (pathologic) }\end{array}$ & Ope & $\begin{array}{c}\text { Resection } \\
\text { margin }\end{array}$ & $\begin{array}{l}\text { OT } \\
\text { (min) }\end{array}$ & $\begin{array}{c}\mathrm{BL} \\
(\mathrm{mL})\end{array}$ & $\begin{array}{l}\text { LOS } \\
\text { (days) }\end{array}$ & Comp \\
\hline Female & 57 & $A$ & $\mathrm{~T} 1 \mathrm{~b}$ & Benign & GB bed & RO & 248 & 50 & 10 & \multirow{10}{*}{$\begin{array}{c}\text { Bile } \\
\text { leakage }\end{array}$} \\
\hline Male & 63 & A & $\mathrm{T} 1 \mathrm{~b}$ & Benign & GB bed & Ro & 296 & 250 & 15 & \\
\hline Female & 72 & A & T1b & T1b & GB bed & Ro & 340 & 150 & 105 & \\
\hline Female & 38 & A & $\mathrm{T} 1 \mathrm{~b}$ & $\mathrm{~T} 1 \mathrm{~b}$ & GB bed & Ro & 186 & 10 & 11 & \\
\hline Male & 82 & A & $\mathrm{T} 1 \mathrm{~b}$ & $\mathrm{~T} 1 \mathrm{~b}$ & GB bed & Ro & 201 & 50 & 17 & \\
\hline Female & 39 & A & $\mathrm{T} 1 \mathrm{~b}$ & $\mathrm{~T} 1 \mathrm{~b}$ & GB bed & Ro & 307 & 50 & 8 & \\
\hline Female & 63 & A & $\mathrm{T} 1 \mathrm{~b}$ & $\mathrm{~T} 1 \mathrm{~b}$ & GB bed & Ro & 197 & 75 & 10 & \\
\hline Male & 65 & A & T2 & T2 & $s 4 b+5+6 a$ & Ro & 300 & 500 & 17 & \\
\hline Male & 69 & A & T2 & $\mathrm{T} 2$ & $S 4 b+5+6 a$ & Ro & 442 & 200 & 34 & \\
\hline Male & 72 & A & T2 & T2 & $S 4 b+5+6 a$ & Ro & 488 & 143 & 24 & \\
\hline
\end{tabular}

GBC: gallbladder carcinoma; LLR: laparoscopic liver resection; Ope: performed operation; OT: operation time; BL: intraoperative blood loss; LOS: postoperative length of hospital stay; Comp: complication; GB bed: LLR of GB bed liver with peri-cystic lymph node and peri-bile duct lymph node dissections; $S 4 b+5+6 a$ : LLR of $S 4 b+5+6 a$ with regional lymph node dissection 
standard deviation unless otherwise noted. Differences in each parameter between the GBT and other groups were evaluated using the Mann-Whitney $U$ test. All analyses were performed using SPSS, version 22.0 (IBM Corp., Armonk, NY, USA). A $P$ value of $<0.05$ (two-tailed) was considered statistically significant.

\section{Operative procedure for GBTs of the fundus/ body suspected to be T1b/T2 GBC}

The patients underwent general anesthesia and were placed in the reverse trendelenburg position. The operating table was tilted to the left or right as necessary to acquire an adequate operative field of view.

The first trocar port was introduced with a minilaparotomy on the umbilicus, and $8-$ to $12-\mathrm{mmHg}$ carbon dioxide pneumoperitoneum was established through this port. This port was also mainly used for the laparoscope. Three other 12-mm ports and one 8-mm port were placed in the upper middle to right abdomen and used to introduce the surgeons' forceps, energy devices (SonoSurg, BiClamp bipolar forceps, and irrigation monopolar electric cautery with soft-mode coagulation), clips, and Cavitron ultrasonic surgical aspirator (CUSA) as well as the assistant's forceps. The Pringle maneuver was not applied.

\section{$S 4 b+5+6 a L L R$}

For $S 4 b+5+6 a$ LLR, the operation was started with liver parenchymal transection on the right edge of the umbilical Glissonian pedicle [Figure 1] after confirming the locations of the GBT and major vessels by intraoperative laparoscopic ultrasonography. If needed, adhesions from a previous surgery were dissected before the ultrasonographic examination and transection. The liver parenchymal transection started with the use of the SonoSurg on the shallow surface of the liver. The BiClamp bipolar forceps, used in a clamp-and-crush manner, and the CUSA were used for deep parenchymal transection far from and near the major vessels, respectively. Small vessels were exposed and sealed with energy devices, clipped or ligated, and finally divided. Hemostasis of bleeding from the transection surface was accomplished by irrigation monopolar electric cautery with soft-mode coagulation or suturing by hand. During the transection on the umbilical line, two or three Glissonian pedicles to $S 4 b$ (G4b) were dissected, encircled, ligated, and divided [Figure 2]. The ischemic demarcation line appeared on the liver surface after division of G4b [Figure 3], showing the left part of the transection line of the resected liver (S4b of $S 4 b+5+6 a)$. According to this line, liver transection was performed from left to right, exposing the hilar plate at the bottom.
The peripheral part of the middle hepatic vein was revealed and divided on the transection plane between S4b and S5 [Figure 4]. When the bottom of the transection line reached the right edge of the hilar plate, the LNs around the bile duct were dissected and the root of the cystic duct was exposed and divided [Figure 5]. Intraoperative frozen section pathology of the stump of the cystic duct confirmed the absence of tumor invasion. The cystic plate including the cystic duct, artery, and LNs was attached to the resected liver. Dissection was then performed from the hepatic duct to right Glissonian pedicle.

During dissection of the right Glissonian pedicle, G5a, G6a, and G5b were exposed and divided [Figure 6]. Liver parenchyma transection was performed according to

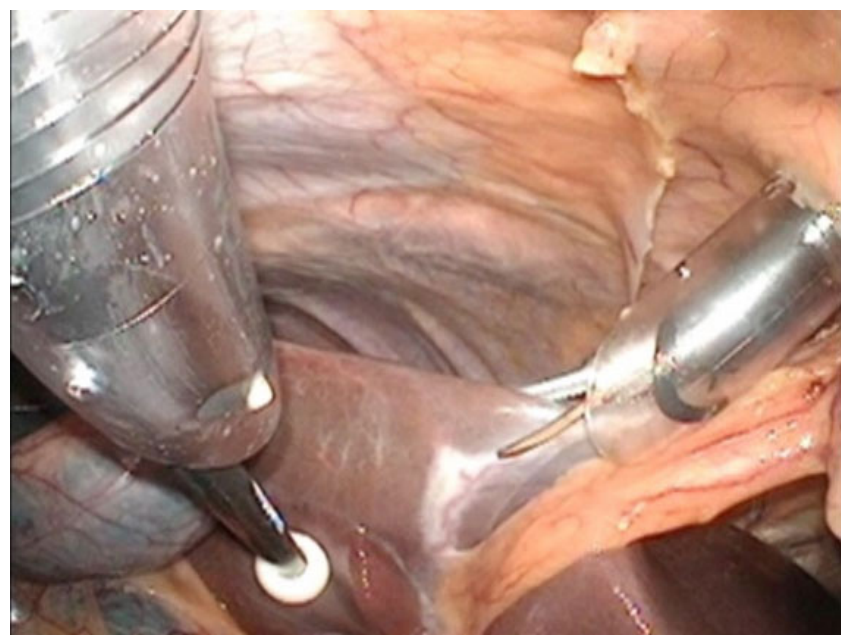

Figure 1: Operative procedure for gallbladder tumor of the fundus/ body suspected to be T1b/T2 gallbladder carcinoma-1 (liver parenchymal transection on right edge of the umbilical plate). For $S 4 b+S 5+S 6 a$ LLR, the operation was started from the liver parenchymal transection on the right edge of the umbilical plate

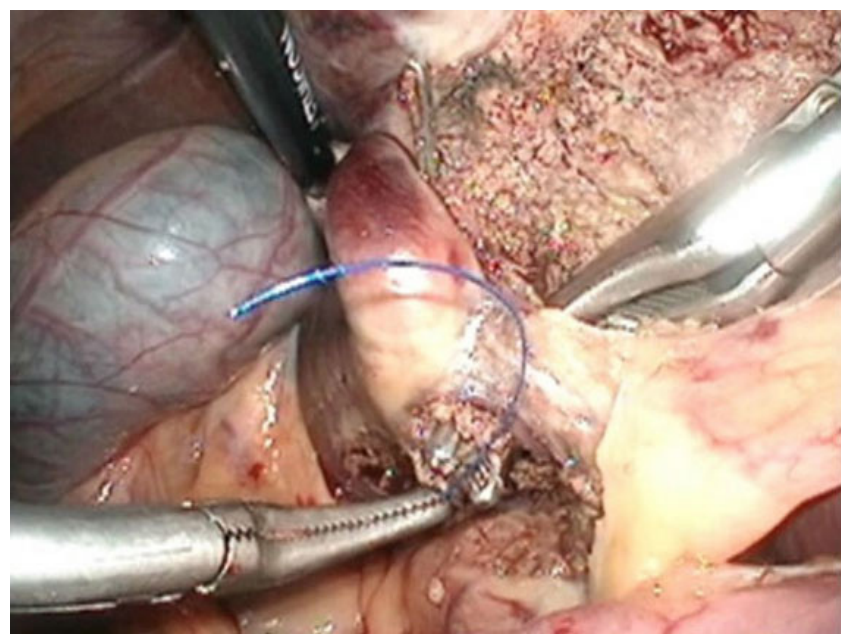

Figure 2: Operative procedure for gallbladder tumor of the fundus/ body suspected to be T1b/T2 gallbladder carcinoma-2 (Glissonian pedicles to S4b). During the transection, the Glissonian pedicles to S4b were dissected, encircled, ligated, and divided 


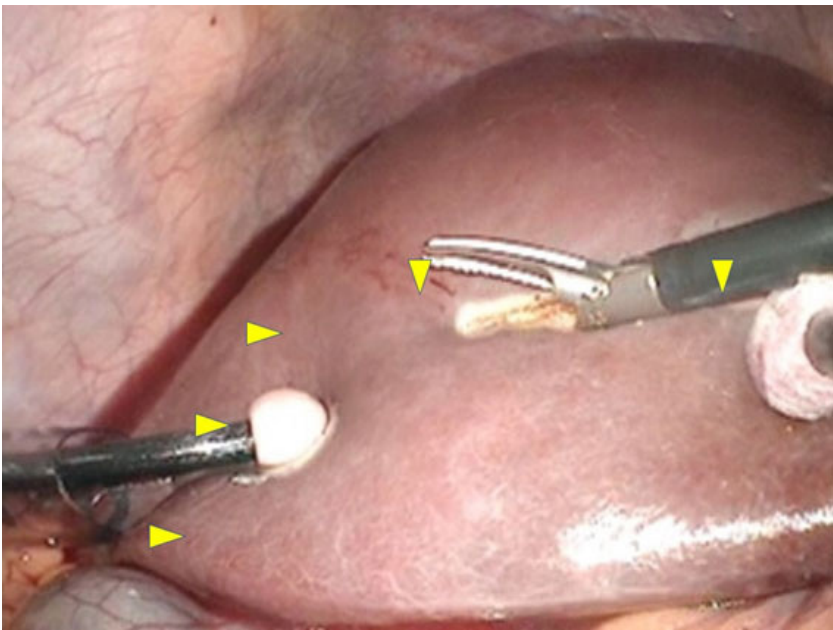

Figure 3: Operative procedure for gallbladder tumor of the fundus/body suspected to be T1b/T2 gallbladder carcinoma-3 (ischemic demarcation line of S4b). The ischemic demarcation line (arrowheads) was observed on the liver surface after division of the Glissonian pedicles to S4b. According to this line, liver transection was performed from left to right, exposing the hilar plate

the ischemic demarcation line on the liver surface that appeared after division of these Glissonian pedicles and exposure of the right part of the transection line of the resected liver (S5+6a of $S 4 b+5+6 a)$ [Figure 7]. The resected liver was extracted in a plastic bag through the umbilical port. Abdominal drainage catheters were routinely placed in the operative area.

\section{GB bed LLR}

For GB bed LLR, the operation started with liver parenchymal transection from the left anterior side (in S4) with a $1-\mathrm{cm}$ surgical margin from the $\mathrm{GB}$ after confirming the locations of the GB bottom in the liver bed, GBT, and major vessels by intraoperative laparoscopic ultrasonography. If needed, adhesions from a previous surgery were dissected before the ultrasonographic examination and transection. The liver parenchymal transection started with use of the SonoSurg on the shallow surface of the liver. The BiClamp bipolar forceps, used in a clamp-and-crush manner, and the CUSA were employed for deep parenchymal transection far from and near the major vessels, respectively. Small vessels were exposed and sealed with energy devices, clipped or ligated, and finally divided. Hemostasis of bleeding from the transection surface was accomplished by irrigation monopolar electric cautery with soft-mode coagulation or suturing by hand. During the transection, small peripheral branches of G4b, middle hepatic vein, G5, and G6a were dissected, ligated, and divided. The liver transection was performed from left to right and ventral to dorsal, reaching the right corner of the hilar plate.

When the transection line reached the right corner of the hilar plate, the LNs around the bile duct were

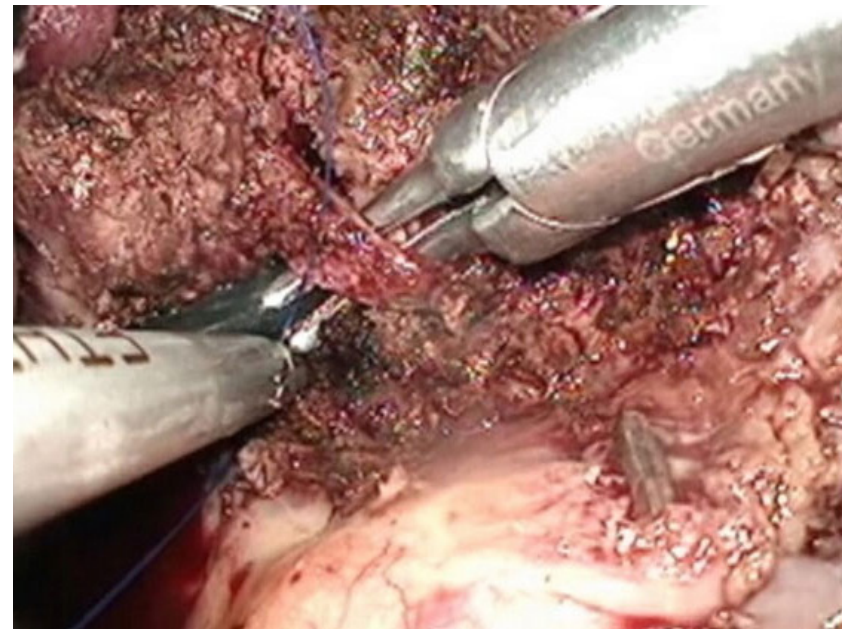

Figure 4: Operative procedure for gallbladder tumor of the fundus/ body suspected to be T1b/T2 gallbladder carcinoma-4 (middle hepatic vein). The peripheral part of the middle hepatic vein was divided on the transection plane

dissected and the root of the cystic duct was exposed and divided. Intraoperative frozen section pathology of the stump of the cystic duct confirmed the absence of tumor invasion. The cystic plate including the cystic duct, artery, and LNs was attached to the resected liver and removed en bloc. The resected liver was extracted in a plastic bag through the umbilical port. Abdominal drainage catheters were routinely placed in the operative area.

\section{Regional LN dissection}

Additional regional $\mathrm{LN}$ dissection was performed after the liver resection when the tumor was confirmed to be T2 GBC. The common bile duct, proper and right hepatic arteries, and portal vein were dissected and taped [Figure 8]. The surrounding tissue including the LNs was resected with the tissues of the common hepatic artery, splenic vein, and posterosuperior surface of the pancreas after performing the Kocher maneuver.

\section{RESULTS}

\section{Conversion, morbidity, and mortality in each group}

Pathological R0 resection was achieved in all 10 patients with GBTs. One patient $(10 \%)$ developed a Clavien-Dindo grade 3 complication (bile leakage) and had a long postoperative LOS (105 days), although no conversions to open procedures or mortality occurred in this group.

Among the 79 patients who underwent PR, 2 (2.5\%) underwent conversions to open procedures and 4 $(5.0 \%)$ developed grade 3 postoperative complications (postoperative ascites, bile leakage, cholecystitis, and 


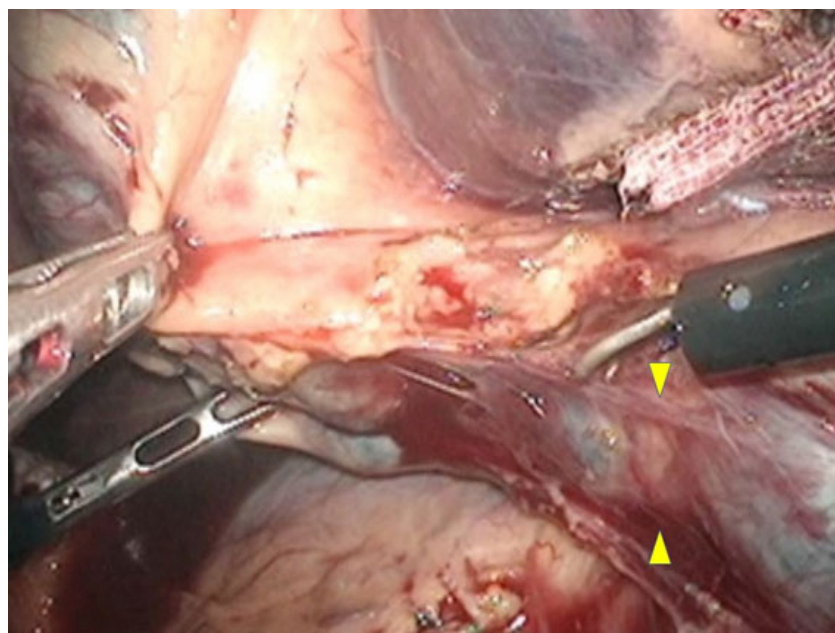

Figure 5: Operative procedure for gallbladder tumor of the fundus/ body suspected to be $\mathrm{T} 1 \mathrm{~b} / \mathrm{T} 2$ gallbladder carcinoma- 5 (cystic plate). When the transection line reached the right side of the hilar plate, the lymph nodes around the bile duct were dissected and the root of the cystic duct (arrowhead) was exposed and divided. The cystic plate including the cystic duct and artery was attached to the resected liver, and dissection from the hepatic duct to the right Glissonian pedicle was performed
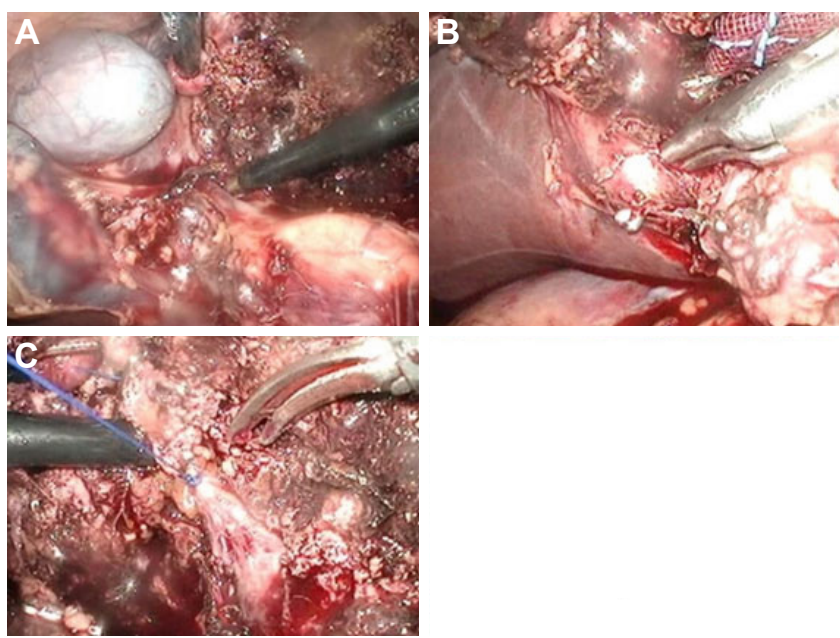

Figure 6: Operative procedure for gallbladder tumor of the fundus/ body suspected to be T1b/T2 gallbladder carcinoma- 6 [Glissonian pedicles, (A) S5a, (B) S6a, and (C) S5b]. During dissection of the right Glissonian pedicles, S5a, S6a, and S5b were exposed and divided

ileus). No mortality occurred.

No conversions or mortality occurred in the LLS, AR, or SAR groups. Two (18.2\%) of 11 patients in the LLS group developed grade 3 postoperative complications (pancreatic juice leakage after pancreaticoduodenectomy in one patient, and postoperative intra-abdominal infectious hematoma after gastrectomy in another patient with protein $S$ deficiency). Two (8.0\%) of 25 patients in the AR group developed grade 3 postoperative complications (ascites and pleural effusion). Two (22.2\%) of 9 patients in the SAR group developed grade 3 postoperative

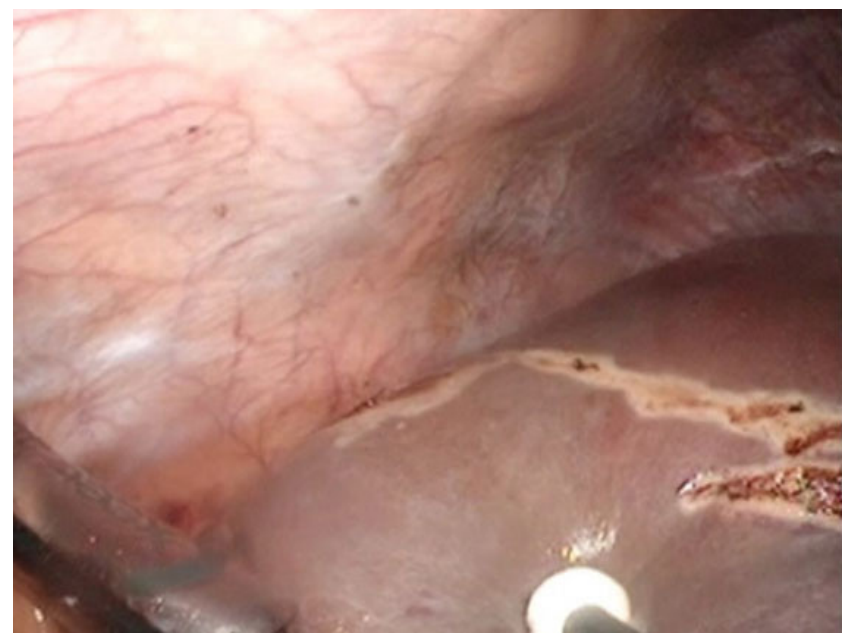

Figure 7: Operative procedure for gallbladder tumor of the fundus/body suspected to be $\mathrm{T} 1 \mathrm{~b} / \mathrm{T} 2$ gallbladder carcinoma-7 (demarcation line after division of Glissonian pedicles, S5a $\mathrm{S} 6 \mathrm{a}$, and S5b). According to the ischemic demarcation line that appeared after division of the Glissonian pedicles, S5a $\mathrm{S} 6 \mathrm{a}$, and S5b, liver parenchymal transection was performed. The resected liver was extracted in a plastic bag through the umbilical port

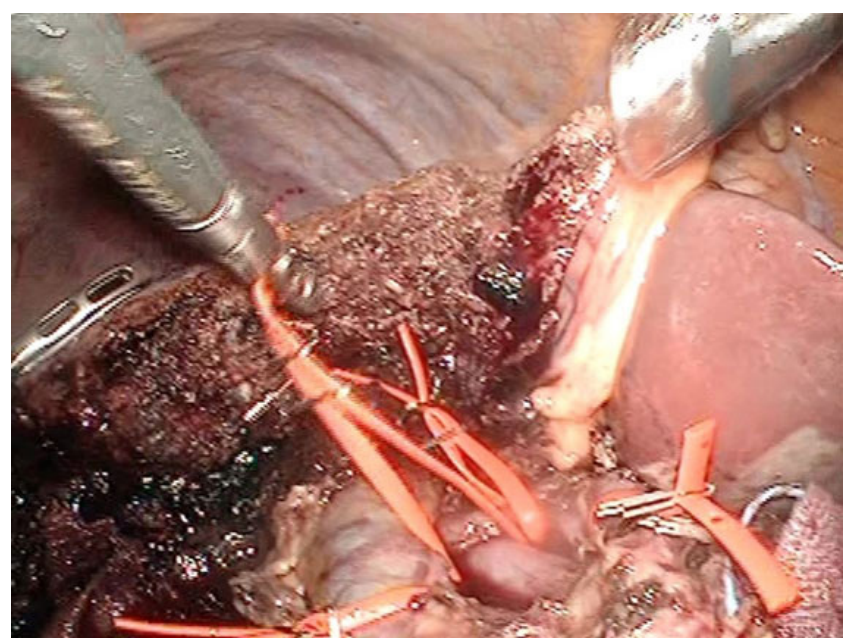

Figure 8: Operative procedure for gallbladder tumor of the fundus/ body suspected to be T1b/T2 gallbladder carcinoma (GBC)-8 [lymph node (LN) dissection]. Regional LN dissection was performed after liver resection when the tumor was pathologically confirmed to be T2 GBC (taped vessels from left to right are the common bile duct, portal vein, right hepatic artery, and proper hepatic artery)

complications (postoperative liver failure for a patient who underwent surgery immediately after the treatment of ruptured esophageal varices, and anastomotic failure of concomitant high anterior rectal resection in the other patient).

No statistically significant differences in the conversion, mortality, or morbidity rates were found among the groups.

\section{OT in each group}

The median OT among all 10 patients with GBTs was $298 \mathrm{~min}$ (range 186-488 $\mathrm{min}$ ), and the mean \pm standard 
deviation was $301 \pm 102 \mathrm{~min}$.

The OT among the 79 patients in the PR group, 11 in the LLS group, 25 in the AR group, and 9 in the SAR group was 245 (84-700) and $292 \pm 140 \mathrm{~min}, 328$ (150-682) and $343 \pm 152 \mathrm{~min}, 458$ (224-848) and 504 $\pm 161 \mathrm{~min}$, and 352 (274-696) and $415 \pm 159 \mathrm{~min}$, respectively.

In the comparison of the OT between patients with GBTs and patients in the other laparoscopic surgery groups (PR, LLS, AR, and SAR) a significant difference was found between the GBT and AR groups [Table 2].

\section{Intraoperative BL in each group}

The BL in patients with GBTs was 109 (10-500) and $148 \pm 145 \mathrm{~mL}$. The $\mathrm{BL}$ in patients who underwent PR, LLS, AR, and SAR was 50 (0/uncountable-3,270) and $278 \pm 556 \mathrm{~mL}, 100(10-516)$ and $166 \pm 182 \mathrm{~mL}, 375$ $(25-3,569)$ and $758 \pm 911 \mathrm{~mL}$, and $705(35-1,920)$ and $821 \pm 794 \mathrm{~mL}$, respectively.

In the comparison of BL between patients with GBTs and patients in the other laparoscopic surgery groups (PR, LLS, AR, and SAR), a significant difference was found between the GBT and AR groups [Table 2].

\section{Postoperative LOS in each group}

The LOS in patients with GBTs was 16 (8-105) and $25 \pm 29$ days. The LOS in the PR, LLS, AR, and SAR groups was 15 (5-254) and $20 \pm 30$ days, 13 (11-52) and $19 \pm 64$ days, 22 (8-44) and $24 \pm 12$ days, and 15 (8-44) and $21 \pm 15$ days, respectively.

No significant differences were found in the LOS between patients with GBTs and patients in the other laparoscopic surgery groups (PR, LLS, AR, and SAR) [Table 2].

Short-term results of LLR of $S 4 b+5+6 a$ (with regional LN dissection) and LLR of GB bed (with peri-cystic LN and peri-bile duct LN dissection)

Of the 10 patients with GBTs, 3 patients with T2 GBC who underwent LLR of $S 4 b+5+6 a$ with regional $L N$ dissection had an OT of 300,442 , and 488 min; BL of 500,200 , and $143 \mathrm{~mL}$; and LOS of 17,34 , and 24 days, respectively. The third patient underwent the surgery, 2 weeks after the first cholecystectomy of severe cholecystitis, for the T2 GBC revealed in the postoperative pathological examination [Table 1].

The other seven patients who underwent LLR of the GB bed with peri-cystic LN and peri-bile duct LN dissections had an OT of 248 (186-340) and $254 \pm$ $61 \mathrm{~min}, \mathrm{BL}$ of $50(10-250)$ and $91 \pm 82 \mathrm{~mL}$, and LOS of 11 (8-105) and $25 \pm 35$ days [Table 1].

\section{DISCUSSION}

Although no differences in LOS were observed, the $\mathrm{BL}$ and OT were significantly lower in the GBT than AR group. Additionally, no differences were observed in the conversion, morbidity, or mortality rate between laparoscopic GBT surgery and conventional LLR of any type. When compared with other types of conventional LLR, the short-term results (OT, BL, and LOS) of all 10 patients with GBTs were comparable with those in the LLS group. Three patients with T2 GBC who underwent LLR of $S 4 b+5+6 a$ with regional LN dissection had perioperative short-term results comparable with those of patients who underwent $A R$, although the number of patients was small. The perioperative short-term results of the other 7 patients who underwent LLR of the GB bed with peri-cystic duct $L N$ and peri-bile duct $L N$ dissections were comparable even with those of patients who underwent PR. LLR of the GB bed or $S 4 b+5+6 a$ with LN dissection was feasible for treatment of GBTs of the body/fundus suspected to be T1b/T2 GBC without cystic duct invasion. Itano et al. ${ }^{[8]}$ reported that laparoscopic surgery for T2 GBC had a comparable OT (368 vs. $352 \mathrm{~min}$ ), significantly smaller BL volume (152 vs. $777 \mathrm{~mL}$ ), shorter LOS (9.1 vs. 21.6 days), and similar morbidity rate (1/15 vs. $3 / 11$ patients) compared with open surgery. Our results are similar to those from their laparoscopic surgeries. LLR has the advantages of a smaller BL volume and shorter LOS in some conditions, such as minor resections of the anterolateral segments. ${ }^{[5]}$ LLR of $S 4 b+5+6 a$

Table 2: Perioperative short-term outcomes of different types of laparoscopic liver resections

\begin{tabular}{|c|c|c|c|c|c|c|}
\hline & \multicolumn{2}{|c|}{ OT $(\min )$} & \multicolumn{2}{|c|}{$\mathrm{BL}(\mathrm{mL})$} & \multicolumn{2}{|c|}{ LOS (days) } \\
\hline GBT $(n=10)$ & $298(186-488)$ & & $109(10-500)$ & & $16(8-105)$ & \\
\hline $\operatorname{PR}(n=79)$ & $245(84-700)$ & NS & 50 (NC -3,270) & NS & $15(5-254)$ & NS \\
\hline $\operatorname{LLS}(n=11)$ & $328(150-682)$ & NS & $100(10-516)$ & NS & $13(11-52)$ & NS \\
\hline $\operatorname{AR}(n=25)$ & $458(224-848)$ & $P<0.001$ & $375(25-3,569)$ & $P<0.05$ & $22(8-44)$ & NS \\
\hline $\operatorname{SAR}(n=9)$ & $352(274-696)$ & NS & $705(35-1,920)$ & NS & $15(8-44)$ & NS \\
\hline
\end{tabular}

Data are shown as median (range). OT: operation time; BL: intraoperative blood loss; LOS: postoperative length of hospital stay; GBT: laparoscopic liver resection with lymph node dissection for gallbladder tumor; PR: laparoscopic partial liver resection; LLS: laparoscopic left lateral sectionectomy of the liver; AR: laparoscopic anatomical resection of the liver (resection of one or more sections, excluding LLS); SAR: laparoscopic small anatomical resection of the liver (resection of less than a full segment); NC: not countable; NS: not significantly different from GBT data; $P<0.001 / P<0.05$ : significantly different from GBT data 
or GB bed with limited LN dissection and no bile duct resection for treatment of GBC is thought to have similar advantages, although such a procedure also has potential disadvantages (risk of tumor cell dissemination and port site recurrence). ${ }^{[11]}$

Liver resection for treatment of T1b/T2 GBC involves $\mathrm{PR}$ of the anterolateral segments, where laparoscopic approaches are easily applied,,$^{[4,5]}$ and the techniques for LN dissection have also been applied in other established procedures. ${ }^{[15,18]}$ Although dissection of the posterosuperior pancreatic and peri-splenic vein LNs is difficult, this dissection can reportedly be easier with the Kocher maneuver ${ }^{[8,9]}$ However, bile duct resection and reconstruction is still a demanding technique with limited reports. ${ }^{[9,16,19,20]}$ It is often required for bile duct invasion by the tumor in patients with T3 GBC or GBC in the neck. Therefore, in the present series, only patients with GBTs suspected to be T1b/T2 GBC in the body/fundus were selected for laparoscopic surgeries with intraoperative pathological examination for confirmation of negative cystic duct tumor invasion. No cases of mismatch of the intraoperative and postoperative pathological results of cystic duct tumor invasion were encountered. Furthermore, an accurate preoperative diagnosis of the tumor depth ( $T$ stages $1 \mathrm{a}, 1 \mathrm{~b}, 2$, and $3^{[12]}$ ) is needed for application of this technique. Itano et al ${ }^{[8]}$ reported that precise preoperative endoscopic ultrasonography led to no underestimation of the preoperative diagnosis regarding tumor invasion into the muscular or subserosal layer in their patients with $\mathrm{T} 1 / \mathrm{T} 2$ cancer. We also attempted to avoid underestimation, which leads to the need for a second operation and/or carcinoma recurrence, rather than overestimation in our series. We observed no cases of underestimation; however, 2 patients had benign (overestimated) lesions, including 1 xanthogranuloma. Overestimation and overapplication of this procedure for benign or Tis/T1a GBC is also a potential problem. However, the drawbacks of laparoscopic cholecystectomy for Tis/T1a GBC include the risk of GB wall perforation and bile leakage contaminated with tumor cells, which may lead to port site recurrence and peritoneal dissemination. ${ }^{[21]}$ These risks could be overcome by combined GB bed resection. Given the fact that this procedure was performed with short-term outcomes comparable with those of laparoscopic LLS or PR, overestimation and overapplication of this procedure might be justified. However, LLR of $S 4 b+5+6 a$ with regional LN dissection, which we applied to the patients with $\mathrm{T} 2 \mathrm{GBC}$, is a more complicated and demanding procedure and was associated with a longer OT and larger BL volume comparable with AR. The application of this procedure is currently limited to patients with proven T2 GBC in our institute.

Itano et $a{ }^{\left[{ }^{[8]}\right.}$ reported that the disease-free and overall survival rates of patients with T2 GBC tended to be superior, although not significantly so, among patients who underwent laparoscopic than open surgery. However, they also mentioned that this observation may have been due to selection bias because their study was a semi-historical control study (the period for the laparoscopic group was from December 2007 to December 2013, and that for the control open group was from June 2003 to May 2011), and patients with more advanced disease might have been selected for the open surgery group before the advent of precise endoscopic ultrasonography examination. They still concluded that the laparoscopic approach for suspected T2 GBC was at least comparable with open surgery in terms of both the surgical and oncological outcomes.

The sample size of the present study was too small to perform a definitive statistical analysis of the short-term outcomes, and concerns regarding tumor dissemination and port site recurrence are still valid when performing laparoscopic procedures with restricted manipulation and instruments. Further studies of laparoscopic surgery for GBC are needed. Nevertheless, this technique could be a good treatment option for GBTs suspected to be T1b/T2 $\mathrm{GBC}$ in the GB body/fundus without invasion of the cystic duct.

\section{DECLARATIONS}

\section{Authors' contributions}

Performed the treatments and wrote this manuscript: M. Isetani

Planned and performed the treatments: Z. Morise

Supervised planning and writing this manuscript: $Z$. Morise, A. Horiguchi

\section{Acknowledgments}

We thank Angela Morben, DVM, ELS, from Edanz Group (www.edanzediting.com/ac) for editing a draft of this manuscript.

\section{Financial support and sponsorship}

None.

\section{Conflicts of interest}

There are no conflicts of interest.

\section{Patient consent}

Patients were fully involved in the treatment decisionmaking process. Informed consent was obtained 
from each patient for both treatment and use of data in the study.

\section{Ethics approval}

The data obtained through the medical record review were managed according to the privacy policy and ethics code of our institute. The surgeries were performed with the permission of our hospital review board.

\section{REFERENCES}

1. Reich H, McGlynn F, DeCaprio J, Budin R. Laparoscopic excision of benign liver lesions. Obstet Gynecol 1991;78:956-8.

2. Katkhouda N, Fabiani P, Benizri E, Mouiel J. Laser resection of a liver hydatid cyst under videolaparoscopy. Br J Surg 1992;79:560-1.

3. Gagner M, Rheault M, Dubuc J. Laparoscopic partial hepatectomy for liver tumor. Surg Endosc 1992;6:97-8.

4. Morise Z, Wakabayashi G. First quarter century of laparoscopic liver resection. World J Gastroenterol 2017;23:3581-8.

5. Wakabayashi G, Cherqui D, Geller DA, Buell JF, Kaneko H, Han HS, Asbun H, O’Rourke N, Tanabe M, Koffron AJ, Tsung A, Soubrane O, Machado MA, Gayet B, Troisi RI, Pessaux P, Van Dam RM, Scatton O, Abu Hilal M, Belli G, Kwon CH, Edwin B, Choi GH, Aldrighetti LA, Cai X, Cleary S, Chen KH, Schön MR, Sugioka A, Tang CN, Herman P, Pekolj J, Chen XP, Dagher I, Jarnagin W, Yamamoto M, Strong R, Jagannath P, Lo CM, Clavien PA, Kokudo N, Barkun J, Strasberg SM. Recommendations for laparoscopic liver resection: a report from the second international consensus conference held in Morioka. Ann Surg 2015;261:619-29.

6. Morise Z, Ciria R, Cherqui D, Chen KH, Belli G, Wakabayashi G. Can we expand the indications for laparoscopic liver resection? A systematic review and meta-analysis of laparoscopic liver resection for patients with hepatocellular carcinoma and chronic liver disease. J Hepatobiliary Pancreat Sci 2015;22:342-52.

7. Gumbs AA, Hoffman JP. Laparoscopic completion radical cholecystectomy for T2 gallbladder cancer. Surg Endosc 2010;24:3221-3.

8. Itano O, Oshima G, Minagawa T, Shinoda M, Kitago M, Abe Y, Hibi T, Yagi H, Ikoma N, Aiko S, Kawaida M, Masugi Y, Kameyama K, Sakamoto M, Kitagawa Y. Novel strategy for laparoscopic treatment of pT2 gallbladder carcinoma. Surg Endosc 2015;29:3600-7.

9. Shirobe T, Maruyama S. Laparoscopic radical cholecystectomy with lymph node dissection for gallbladder carcinoma. Surg Endosc 2015;29:2244-50.

10. Lee JM, Kim BW, Kim WH, Wang HJ, Kim MW. Clinical implication of bile spillage in patients undergoing laparoscopic cholecystectomy for gallbladder cancer. Am Surg 2011;77:697-701.

11. Yamamoto H, Hayakawa N, Kitagawa Y, Katohno Y, Sasaya T, Takara D, Nagino M, Nimura Y. Unsuspected gallbladder carcinoma after laparoscopic cholecystectomy. $J$ Hepatobiliary Pancreat Surg 2005; 12:391-8.

12. Oh TG, Chung MJ, Bang S, Park SW, Chung JB, Song SY, Choi GH, Kim KS, Lee WJ, Park JY. Comparison of the sixth and seventh editions of the AJCC TNM classification for gallbladder cancer. $J$ Gastrointest Surg 2013;17:925-30.

13. Fong Y, Jarnagin W, Blumgart LH. Gallbladder cancer: comparison of patients presenting initially for definitive operation with those presenting after prior noncurative intervention. Ann Surg 2000;232:557-69.

14. Shibata K, Uchida H, Iwaki Kai S, Ohta M, Kitano S. Lymphatic invasion: an important prognostic factor for stages T1b-T3 gallbladder cancer and an indication for additional radical resection of incidental gallbladder cancer. World J Surg 2009;33:1035-41.

15. Cai J, Wei D, Cao CF, Zhang CS, Zhang H, Zhao T. A prospective randomized study comparing open versus laparoscopy-assisted D2 radical gastrectomy in advanced gastric cancer. Dig Surg 2011;28:331-7.

16. Gumbs AA, Hoffman JP. Laparoscopic radical cholecystectomy and Roux-en-Y choledochojejunostomy for gallbladder cancer. Surg Endosc 2010;24:1766-8

17. Hu JB, Sun XN, Xu J, He C. Port site and distant metastases of gallbladder cancer after laparoscopic cholecystectomy diagnosed by positron emission tomography. World $J$ Gastroenterol 2008;14:6428-31.

18. KendrickML,CusatiD. Totallaparoscopicpancreaticoduodenectomy: feasibility and outcome in an early experience. Arch Surg 2010;145:19-23.

19. Asbun HJ, Harada E, Stauffer JA. Tips for laparoscopic pancreaticoduodenectomy. $J$ Hepatobiliary Pancreat Sci 2016;23:E5-9.

20. Abbas HM, Yassin NA, Ammori BJ. Laparoscopic resection of type I choledochal cyst in an adult and Roux-en-Y hepaticojejunostomy: a case report and literature review. Surg Laparosc Endosc Percutan Tech 2006; 16:439-44.

21. Cavallaro A, Piccolo G, Panebianco V, Lo Menzo E, Berretta M, Zanghi A, Di Vita M, Cappellani A. Incidental gallbladder cancer during laparoscopic cholecystectomy: managing an unexpected finding. World J Gastroenterol 2012;18:4019-27. 